\title{
Anopheles pseudowillmori is the predominant malaria vector in Motuo County, Tibet Autonomous Region
}

\author{
Wu Song1,3, Pan Jia-Yun1, Wang Xue-Zhong², Zhou Shui-Sen ${ }^{1}$, Zhang Guo- \\ Qing1, Liu Qian ${ }^{3}$ and Tang Lin-Hua*1
}

Address: ${ }^{1}$ National Institute of Parasitic Diseases, Chinese Center for Disease Control and Prevention, Shanghai, PR China, ${ }^{2}$ Yunnan Institute of Parasitic Diseases, Puer, PR China and ${ }^{3}$ School of Integrated Traditional and Western Medicine, Anhui College of Chinese Traditional Medicine, Hefei, PR China

Email: Wu Song - jswu167@126.com; Pan Jia-Yun - jypan15@yahoo.com.cn; Wang Xue-Zhong - xuezhongw@hotmail.com; Zhou ShuiSen - ccdczss@sh163.net; Zhang Guo-Qing - ahmuzgq@163.com; Liu Qian - lq254@126.com; Tang Lin-Hua* - ipdtlh@sh163.net

* Corresponding author

Published: 16 March 2009

Malaria Journal 2009, 8:46 doi:10.1 /86//475-2875-8-46

This article is available from: http://www.malariajournal.com/content/8/l/46

(c) 2009 Song et al; licensee BioMed Central Ltd.

This is an Open Access article distributed under the terms of the Creative Commons Attribution License (http://creativecommons.org/licenses/by/2.0), which permits unrestricted use, distribution, and reproduction in any medium, provided the original work is properly cited.
Received: 9 October 2008

Accepted: 16 March 2009

\begin{abstract}
Background: Malaria is endemic in Linzhi Prefecture in the Tibet Autonomous Region (TAR), but the vector for malaria transmission had never been identified.

Methods: Adult Anopheles spp. were collected in Motuo County, Linzhi Prefecture on the SinoIndian border in July and August, 2007. Multiplex PCR was adopted for species identification, and a nested PCR approach was used to detect sporozoites in the salivary glands of the mosquitoes.

Results: 3,675 mosquitoes of the Anopheles maculatus group were collected and processed for species identification. Among them, 3,602 (98.0\%) were Anopheles pseudowillmori and 73 (2.0\%) were Anopheles willmori. The Plasmodium vivax SSUrDNA fragment was amplified in two of 360 pooled An. pseudowillmori samples.

Conclusion: The local An. maculatus group comprises the species An. pseudowillmori and An. willmori. Anopheles pseudowillmori is considered the sole malaria vector in Motuo County in Linzhi Prefecture.
\end{abstract}

\section{Background}

Linzhi Prefecture is located in the south-eastern part of the Tibet Autonomous Region (TAR) of China. Motuo County in south of Linzhi Prefecture had a total population of 10,019 in 2006 and shares borders with both India and Myanmar. A total of 2,459 malaria cases were reported from Linzhi Prefecture between 1986 and 2004. Most of these infections were attributed to Plasmodium vivax, and 2,441 (99.3\%) of the cases originated from Motuo County [1]. In 2005 and 2006, the annual malaria incidence rates (IR) in Motuo County were 56.8 and 69.4 cases per 10,000 persons, respectively. Also in 2005, a malaria outbreak was reported from Bayi Town out of Motuo County, indicating an increasing malaria threat.

Up to the present day, Motuo County is inaccessible by car, and most villages can only be reached by foot. Although malaria appears to be a considerable public health problem in Motuo County, only few studies focused on this area, and the local malaria vector(s) 
remained elusive. This can be attributed both to the lack of local health workers, and to the challenging geographical conditions.

Studies conducted in 2006 established that Anopheles maculatus s.l. is the dominant Anopheles taxon in Motuo County (unpublished). The An. maculatus Theobald group belongs to the Neocellia series of the subgenus Cellia and is distributed throughout the Oriental region [2]. Globally, nine members of the An. maculatus group have been described $[3,4]$. Five among them occur in China. These are An. maculatus s.s., An. willmori, An. pseudowillmori, Anopheles sawadwongporni and Anopheles dravidicus [5,6]. Anopheles maculatus s.l. and related species have long been recognized as important malaria vectors in Malaysia [7], Thailand [8] and the Philippines [9]. Anopheles maculatus s.l. infected with Plasmodium were also detected in Yunnan Province, south-west China [10].

The aim of the present study was to elucidate the significance of An. maculatus s.l. for malaria transmission in Motuo County, thus providing the basis for the development and implementation of a locally adapted integrated malaria control strategy.

\section{Methods}

\section{Study sites and period}

Motuo County stretches across the lower reaches of the Brahmaputra River between $27^{\circ} 36^{\prime}$ and $29^{\circ} 50^{\prime} \mathrm{N}$ latitude and $93^{\circ} 42^{\prime}$ and $96^{\circ} 36^{\prime} \mathrm{E}$ longitude. The altitude of the mountainous county ranges between 700 and 2,100 m above sea level (mean: 1,200 $\mathrm{m}$ ) and the average annual temperature is $16.1{ }^{\circ} \mathrm{C}$. Almost all malaria-endemic villages are scattered along the Brahmaputra River. These villages are mostly inhabited by members of the Zang, Menba and Luoba nationalities.

The study was implemented during the peak malaria transmission season from July to September 2007 in three villages(Figure 1) where the malaria incidence was high in previous years, namely Yadong $\left(29^{\circ} 32^{\prime} \mathrm{N}, 95^{\circ} 33^{\prime} \mathrm{E}\right)$, Dexin $\left(29^{\circ} 33^{\prime} \mathrm{N}, 95^{\circ} 30^{\prime} \mathrm{E}\right)$ and Madi $\left(29^{\circ} 37^{\prime} \mathrm{N}, 95^{\circ} 41^{\prime} \mathrm{E}\right)$ near the Sino-Indian border. The inhabitants of these villages live in houses and huts grouped into hamlets scattered across the village territory, usually $1-3 \mathrm{~km}$ apart from each other and only accessible by foot.

\section{Sample collection}

Cow-baited traps (CBT), human-baited net traps (HBNT) and CDC light traps (New Standard Miniature Light Traps $5126 \mathrm{~V} 150 \mathrm{~mm}$ ) were set up to collect adult mosquitoes between 21:00 and 01:00, or between 21:00 to 06:00. Each morning, the trapped mosquitoes were counted and identified according to morphological criteria using the key developed by Lu BL [5]. According to Manguin et al.

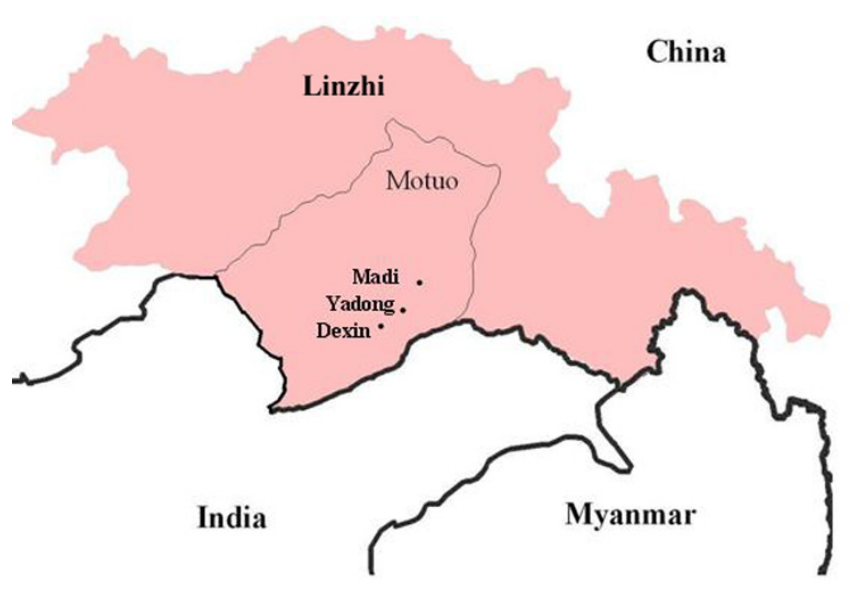

Figure I

The map of relative study sites.

[2], the morphological identification of adult members of the An. maculatus group is error-prone due to overlapping characteristics; therefore, the collected samples were morphologically classified by species complex rather than species. Following the classification, the mosquitoes were killed by chloroform and dried on silica-gel, and subsequently transported to the laboratory where they were stored at $-20^{\circ} \mathrm{C}$ pending DNA extraction.

\section{DNA extraction}

All An. maculatus s.l. adults were individually registered. Subsequently, about $70 \%$ An. maculatus s.l. DNA was extracted from the legs of each individual for species identification by multiplex PCR, and pooled samples each comprising 10 thoraxes of the same species were homogenized to extract DNA for sporozoites identification as described by Li et al. [11] and Sun et al. [12]. DNA extraction followed the protocol presented by Collins et al. [13], and the quality of the isolated DNA was assessed by measuring the proportional optical density (OD) at 260/280 $\mathrm{nm}$.

\section{Species identification by multiplex PCR}

3,675 An. maculatus s.l. specimens underwent species identification by multiplex PCR. A protocol capable of distinguishing five members of the An. maculatus s.l. was adapted from Ma et al. [6], with primers designed based on sequence variations in the second internal transcribed spacer (ITS2) of the ribosomal DNA (rDNA). Anopheles pseudowillmori, An. maculatus s.s., An. willmori, An. dravidicus and An. sawadwongporni are characterized by 119 bp, 186 bp, 231 bp, 327 bp and 406 bp fragments, respectively. Well-characterized DNA samples were used as positive controls. The amplified products were spread by electrophoresis on a $2 \%$ agarose gel, stained with ethidium bromide, and visualized using the Gel Imaging Sys- 
tem (Alpha Imager HP, USA). A subsample of the PCR products was cloned and sequenced for confirmation.

\section{Detection of $P$. vivax by nested PCR}

The DNA of 3,600 An. pseudowillmori (= 360 pooled samples) and 70 An. willmori (= 7 pooled samples) thoraxes was extracted according to the Collins method [13]. For the nested PCR, the following primers described by Snounou et al. [14] were used: the Plasmodium specific SSUrDNA primers rPLU5 (5'-CCTGTTGTTGCCTTAAACTTC-3') and rPLU6 (5'-TTAAAATTGTTGCAGTTAAAACG-3'), and the Plasmodium vivax species-specific SSUrDNA primers rVIV1 (5'-CGCTTCTAGCTTAATCCACATAACTGATAC-3') and rVIV2 (5'-ACTTCCA AG CC GAAGCAAAGAAAGTCCTTA-3'). DNA amplification followed the protocol of Tassanakajon [15]. Specifically, the first amplification cycle was performed using the primers rPLU5 and rPLU6 in $50 \mu \mathrm{l}$ reaction mixture containing 1 $\mu \mathrm{l}$ DNA template. For the second cycle, $1 \mu \mathrm{l}$ of the firstround product was used as template for the primers rVIV1 and rVIV2. Pooled samples were considered positive if a 121 bp fragment was obtained in the second PCR cycle. All products were cloned, sequenced and blasted in NCBI BLAST http://blast.ncbi.nlm.nih.gov/Blast.cgi.

\section{Results \\ Anopheline species complex and species identification}

During the study period, a total of 5,345 adult anopheline mosquitoes were collected by HBNTs, CBTs and CDC light traps. Among them, An. maculatus s.l. was the predominant taxon representing $97.1 \%(5,190)$ of the total number of collected anopheline mosquitoes. An. peditaeniatus accounted for 2.9\%(155). A total of 3,675 morphologically-identified An. maculatus s.l. were randomly selected for species identification by multiplex PCR. The 231 bp fragment corresponding to An. willmori and the 119 bp fragment associated with An. pseudowillmori were amplified 3,602 (98.0\%) and $73(2.0 \%)$ times, respectively (Figure 2). Cloning and sequencing of the $231 \mathrm{bp}$ fragment yielded the following sequence: 5'-CT GCAGGG CACATGAACACCGTTGAACGCATATTGCGCATCGGA C GCTTCAACCCGACCGATGCACACAT CCTTGAGTGC CT ACCAAGTTATCTATTTTCTCCTACCAAACTGACCGTCC CATCCCCGTGATG GGCTGTCGCAGCATGGCGTGC TC GGACCCGCATCTGTCGGGACCGTGGGCGTTGATAG T GA GAGTGCTATTAT AACGAATGGGGTTACACTATGG G GC-3'. The sequence of the 119 bp fragment was: 5'GAACTGCAGGACACATGAACACCGTTGAACGCATATTGCGCA TCGGACGATTCACCCGACCGATGCACACATCCTTGAGTGC CT AC TCAGT TATCT TATATGC CC ATAC CAGACTAGAC-3'. The NCBI Blast revealed a homology of $98 \%$ between the 231 bp fragments and the An. willmori-specific sequence AF512552.1. The respective value for the $119 \mathrm{bp}$ fragment and An. pseudowillmori sequence AF512550.1 was $97 \%$.

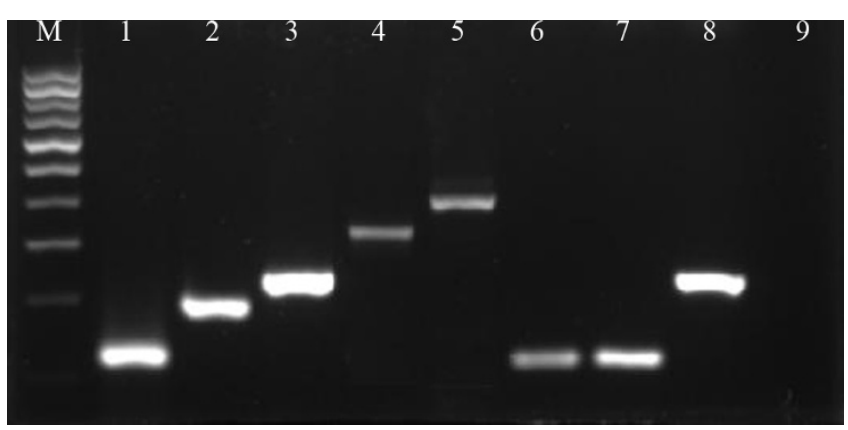

Figure 2

Anopheles maculatus s.l. species identification by PCR. Ethidium bromide-stained PCR products from five species belonging to An. maculatus s.l. as well as positive samples collected in Motuo County, TAR after electrophoresis on agarose gel. Lane M: I00 bp ladder; lane I: An. pseudowillmori; lane 2: An. maculatus s.s.; lane 3: An. willmori; lane 4: An. dravidicus; lane 5: An. sawadwongporni; lanes 6-8: Anopheles spp. collected in Motuo County, TAR; lane 9: negative control.

\section{Sporozoite detection}

A 121 bp fragment was amplified from two of the 360 pooled An. pseudowillmori samples (Figure 3). Cloning and sequencing of the product confirmed the presence of the $P$. vivax SSUrDNA fragment which was found to be identical with the previously reported sequence: 5'ACTTCCAAGCCGAAGCAAAGAAAGTCCTTAAAAAGAAT CATTTTAATTAAAAGAACACATAATAGCAAAA TG CG CA CAAAGTCGATACGAAGTATCAGTTATGTGGATTA AGC T AGAAGCG-3' (AF145335, nt 520 - 640). The amplifica-

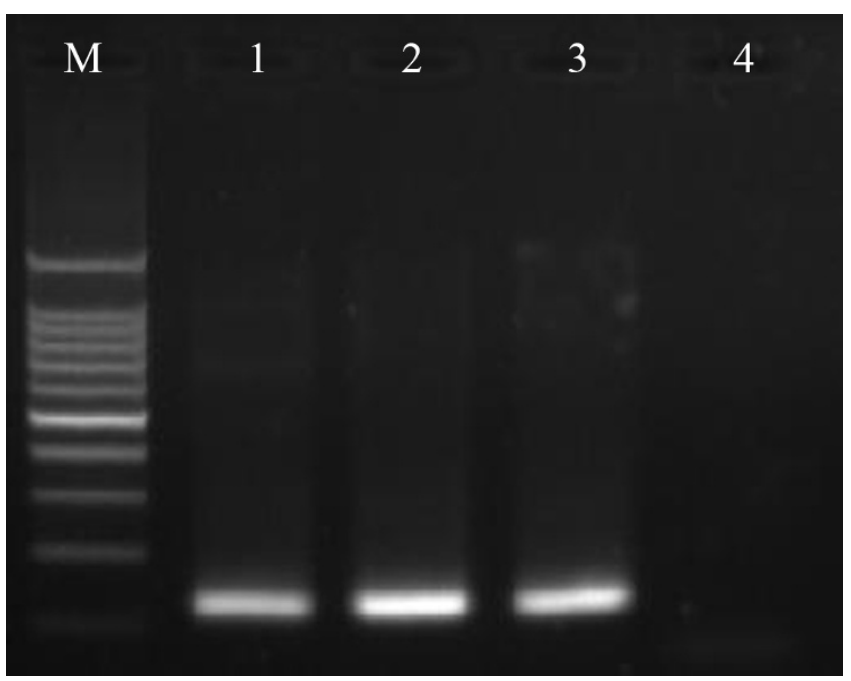

Figure 3

Sporozoite detection by PCR. Ethidium bromide-stained PCR products from the nested PCR approach for sporozoite detection. Lane M: 100 bp ladder; lane I: positive control; lanes 2-3: pooled samples from Motuo County, TAR; lane 4: negative control. 
tion of seven pooled An. willmori samples yielded no positive results.

\section{Discussion}

This is the first investigation aiming at identifying the malaria vector(s) in Motuo County of Linzhi Prefecture, TAR. According to the present results, the Anopheles spp. population in Motuo County almost exclusively consists of An. maculatus s.l. with Anopheles peditaeniatus accounting for the remainder. A previous study [16] reported that Anopheles sinensis, one of the major malaria vectors in central China, also occurred in the TAR. However, the present study failed to consolidate this claim. Although an experimental study [17] had confirmed that the sporozoites of Plasmodium cynomolgi could develop in An. peditaeniatus and that this mosquito could also transmit them to monkeys, there currently is no direct evidence for the transmission of human malaria by this species. Thus, An. maculatus s.l. can be regarded as the sole local malaria vector.

The study further showed that the An. maculatus group in Motuo County comprises the two species An. willmori and An. pseudowillmori. Anopheles willmori is one of the primary malaria vectors in Nepal [18] and has previously been reported to occur in the TAR [5]. However, its local relevance for malaria transmission might be limited regarding its smaller abundance compared to An. pseudowillmori. The latter has been established as a malaria vector in northwest Thailand along the border with Myanmar $[19,20]$ but has not been recorded in the TAR before. Dong et al. [21], however, reported a clear relationship between the seasonal abundance variation of An. pseudowillmori and malaria IR in a mountainous area of Yunnan Province in the south of the TAR.

The detection of sporozoites in the salivary glands of Anopheles spp. conclusively identifies them as malaria vectors. Traditionally, sporozoite infection rates were determined by dissection and examination of salivary glands of individual mosquitoes under a light microscope, a time consuming and labor intensive approach. Therefore, alternative sporozoite detection methods had been developed in recent years: enzyme-linked immunosorbent assay (ELISA) $[7,9,22-24]$ detecting the circumsporozoite protein (CSP), and different approaches based on the PCR technique $[25,26]$ aiming at amplifying specific DNA sequences. It appears that the detection of Plasmodium falciparum and $P$. vivax specific DNA sequences in mosquitoes by PCR has a higher sensitivity compared to ELISA tests [27].

In this study, a nested PCR approach aiming at the identification of the SSUrDNA of $P$. vivax sporozoites had been selected to identify malaria vectors. A conventional PCR test can detect as few as 10 sporozoites per salivary gland, making it a useful tool for screening small numbers of Anopheles spp. [15]. The sensitivity of the employed nested PCR test is as low as three sporozoites [11], and the pooling of samples allows screening of large samples [28]. In our study, pooling samples $[11,12]$ and screening them by nested PCR $[14,15]$ resulted in the identification of $P$. vivax SSUrDNA in salivary glands of two pooled An. pseudowillmori samples, thus establishing that An. pseudowillmori is the main malaria vector in this area.

\section{Conclusion}

The composition of the local anopheline population in Motuo County at the Sino-Indian border consists mostly of members of the An. maculatus group, with An. pseudowillmori being much more abundant than An. willmori. Anopheles pseudowillmori has been identified as the local malaria vector.

\section{Competing interests}

The authors declare that they have no competing interests.

\section{Authors' contributions}

WS performed field and laboratory work and wrote the manuscript. PJY, WXZ and ZSS performed the field work. ZGQ, LQ performed laboratory work. TLH was involved in all stages of this study.

\section{Acknowledgements}

The authors thank Prof. Ma Ya-Jun from the Second Military Medical University of China in Shanghai for providing confirmed DNA samples of five species of the An. maculatus group. The smooth collaboration with the staff of the Linzhi Prefecture CDC and the Motuo County CDC is acknowledged, and we are grateful to Zhuoma Yang-jin, Hu Yong-Hong, Jiang WeiKang, Hu Song-Lin, Xu Hui-Mei, Ciren Quzh and Zhang Wei for their assistance in the field. We thank Peter Steinmann from Swiss Tropical Institute for assistance with the English.

\section{References}

I. Luo S, Hu YH, Hu SL, Zhen N, Li CC: [An epidemiological analysis on malaria in Linzhi District of Tibet in 1986-2004]. Chin J Parasitol Dis 2005, I 2:457-459.

2. Manguin S, Garros C, Dusfour I, Harbach RE, Coosemans M: Bionomics, taxonomy, and distribution of the major malaria vector taxa of Anopheles subgenus Cellia in Southeast Asia: An updated review. Infect Genet Evol 2007, 8:489-503.

3. Harbach RE: The classification of genus Anopheles (Diptera: Culicidae): a working hypothesis of phylogenetic relationships. Bull Entomol Res 2004, 94:537-553.

4. Walton C, Somboon P, O'Loughlin SM, Zhang S, Harbach RE, Linton YM, Chen B, Nolan K, Duong S, Fong MY, Vythilingum I, Mohammed ZD, Trung HD, Butlin RK: Genetic diversity and molecular identification of mosquito species in the Anopheles maculatus group using the ITS2 region of rDNA. Infect Genet Evol 2007, 7:93-102.

5. LU BL: Fauna Sinaca, Insecta, Diptera: Culicidae II Volume 9. Beijing china, Science Press; 1997.

6. Ma YJ, Li SZ, Xu J: Molecular identification and phylogeny of the Maculatus group of Anopheles mosquitoes (Diptera: Culicidae) based on nuclear and mitochondrial DNA sequences. Acta Trop 2006, 99:272-280.

7. Reid JA: Anopheline mosquitoes of Malaya and Borneo. Stud Inst Med Res Malaysia 1968, 31: I-520. 
8. Rattanarithikul R, Konishi E, Linthicum KJ: Detection of Plasmodium vivax and Plasmodium falciparum circumsporozoite antigen in anopheline mosquitoes collected in southern Thailand. Am J Trop Med Hyg 1996, 54(2): | |4-|2|.

9. Torres EP, Foley DH, Saul A: Ribosomal DNA sequence markers differentiate two species of the Anopheles maculatus (Diptera: Culicidae) complex in the Philippines. J Med Entomol 2000, 37:933-937.

10. Zhou HN, Zhang ZX, Curtis C, Hill N, Li CF, Chao W: [Evaluation of the enzyme-linked immunosorbant assay in detecting circumsporozoite protein of anopheline vectors in Yunnan]. Chin J Parasitol Parasit Dis 2004, 22:227-230.

11. Li FW, Niu C, Ye BH: Nested polymerase chain reaction in detection of Plasmodium vivax sporozoites in mosquitoes. Chinese Medical Journal 200 I, I I 4:654-657.

12. Sun QW, Zhu HM, Lu L, Gu ZC, Cheng X, Fang Y: [Restudy on a model for estimating the sporozoite infection rate in mosquitoes using pooled sampling]. Chin J Parasitol Parasit Dis 2002, 20:35I-353.

13. Collins FH, Mendez MA, Rasmussen MO, Mehaffey PC, Besansky NJ, Finnerty $\mathrm{V}$ : A ribosomal RNA gene probe differentiates member species of the Anopheles gambiae complex. Am J Trop Med Hyg 1987, 37(I):37-4I.

14. Snounou G, Pinheiro L, Goncalves A, Fonseca L, Dias F, Brown KN, do Rosario VE: The importance of sensitive detection of malaria parasites in the human and insect hosts in epidemiological studies, as shown by the analysis of field samples from Guinea Bissau. Trans R Soc Trop Med Hyg 1993, 87:649-653.

15. Tassanakajon A, Boonsaeng V, Wilairat P, Panyim S: Polymerase chain reaction detection of Plasmodium falciparum in mosquitoes. Trans R Soc Trop Med Hyg 1993, 87:273-275

16. Editorial Committee of malaria control and research in China: Malaria control and research in China Beijing, People's Medical Publishing House; 1991.

17. Collins WE, Warren M, Galland GG: Studies on infections with the Berok strain of Plasmodium cynomolgi in monkeys and mosquitoes. J Parasitol 1999, 85:268-272.

18. Pradhan SP, Shrestha SL, Vaidya RG: Malaria transmission in high mountain valleys of west Nepal including the first record of Anopheles willmori (James) as a third vector of malaria. Nepal Med Assoc 1970, 8:89-97.

19. Green CA, Rattanarithikul R, Pongparit S, Sawadwongporn P, Baima $V$ : A newly-recognized vector of human malarial parasites in the Oriental region, Anopheles (Cellia) pseudowillmori (Theobald, 1910). Trans R Soc Trop Med Hyg 1991, 85:35-36.

20. Green CA, Rattanarithikul R, Charoensub A: Population genetic confirmation of species status of the malaria vectors Anopheles willmori and An. pseudowillmori in Thailand and chromosome phylogeny of the Maculatus group of mosquitoes. Med Vet Entomol 1992, 6:335-34I.

21. Dong XS, Zhou HL, Bi Y, Dong LM: [The relationship between Anopheles maculatus geographic distribution, ecological features and malaria in Yunnan province]. Acta Parasitol Med Entomol Sin 1996, 3:100-105.

22. Trung HD, Van Bortel W, Sochantha T, Keokenchanh K, Quang NT, Cong LD, Coosemans M: Malaria transmission and major malaria vectors in different geographical areas of Southeast Asia. Trop Med Int Health 2004, 9:230-237.

23. Benet A, Mai A, Bockarie F, Lagog M, Zimmerman P, Alpers MP, Reeder JC, Bockarie MJ: Polymerase chain reaction diagnosis and the changing pattern of vector ecology and malaria transmission dynamics in Papua New Guinea. The American journal of tropical medicine and hygiene 2004, 7 I (3):277-284.

24. Cuamba N, Choi KS, Townson H: Malaria vectors in Angola: distribution of species and molecular forms of the Anopheles gambiae complex, their pyrethroid insecticide knockdown resistance (kdr) status and Plasmodium falciparum sporozoite rates. Malar J 2006, 5:2.

25. Stoffels JA, Docters van Leeuwen WM, Post RJ: Detection of Plasmodium sporozoites in mosquitoes by polymerase chain reaction and oligonucleotide rDNA probe, without dissection of the salivary glands. Med Vet Entomol 1995, 9:433-437.

26. Moreno M, Cano J, Nzambo S, Bobuakasi L, Buatiche JN, Ondo M, Micha F, Benito A: Malaria Panel Assay versus PCR: detection of naturally infected Anopheles melas in a coastal village of Equatorial Guinea. MalarJ 2004, 3:20.
27. Vythilingam I, Nitiavathy K, Yi P, Bakotee B, Hugo B, Singh B, Wirtz RA, Palmer K: A highly sensitive, nested polymerase chain reaction based method using simple dna extraction to detect malaria sporozoites in mosquitos. Southeast Asian J Trop Med Public Health 1999, 30(4):63I-635.

28. Zhou HY, Zhu GD, Jin XL, Li JL, Wang WM, Cao J, Gu YP, Gao Q: [Detection of Plasmodium sporozoites in mosquitoes by nested-PCR]. Chin J Schisto Control 2006, I8:365-368.
Publish with BioMed Central and every scientist can read your work free of charge

"BioMed Central will be the most significant development for disseminating the results of biomedical research in our lifetime. "

Sir Paul Nurse, Cancer Research UK

Your research papers will be:

- available free of charge to the entire biomedical community

- peer reviewed and published immediately upon acceptance

- cited in PubMed and archived on PubMed Central

- yours - you keep the copyright

Submit your manuscript here:

http://www.biomedcentral.com/info/publishing_adv.asp
BioMedcentral 